\title{
Educación musical, epistemocracia y postcovid-19
}

\section{Music education, epistemocracy and postcovid-19}

José A. Rodríguez-Quiles

kiles@ugr.es

Departamento de Didáctica de la Expresión Musical, Plástica y Corporal

Universidad de Granada

Granada, España

ORCID: https://orcid.org/0000-0003-2490-0540

doi: 10.7203/LEEME.47.17550

Recibido: 08-06-2020 Aceptado: 18-08-2020. Contacto y correspondencia: José A. Rodríguez-Quiles y García, Departamento de Didáctica de la Expresión Musical, Plástica y Corporal, Universidad de Granada, Campus de Cartuja, s/n, C.P. 18071 Granada. España.

\section{Resumen}

Como área académica, la Educación Musical está obligada a mantener un singular equilibrio en el seno de la universidad española; situación que ha corrido paralela a la suerte de la música como materia curricular desplazada a los márgenes, tanto en Educación Primaria como Secundaria. Este estatus de precariedad permanente se ha llegado a naturalizar como consecuencia de la imposición de unas reglas de juego por parte de las áreas hegemónicas, a lo que hay ahora que unir las consecuencias sobrevenidas con motivo de la pandemia por la covid-19 y que sólo contribuirá a acentuar más la brecha entre áreas académicas. En el marco de una pedagogía crítica y con la ayuda de la teoría del performativo desde un posicionamiento educativo, en este ensayo se reflexiona sobre las causas de esta situación con el objetivo de poner de manifiesto las tensiones a las que se ve sometida la Educación Musical española, impidiéndosele su avance en el contexto del Espacio Europeo de Educación Superior. Para ello, resulta urgente reconocer y aceptar las diferencias e imaginar otras formas de pensar y hacer universidad desde la diversidad de las áreas de conocimiento y no desde la imposición del pensamiento hegemónico actual. Es intención del presente trabajo incitar al debate científico desde un área que se pretende desterrar al extrarradio del conocimiento como consecuencia de unas políticas educativas segregadoras.

Palabras clave: Educación musical; formación del profesorado de música; precariedad; Espacio Europeo de Educación Superior.

\begin{abstract}
As an academic area, Music Education is obliged to maintain a singular balance within the Spanish university; situation that has run parallel to the fate of music as a curricular subject displaced to the margins, both in Primary and Secondary Education.This status of permanent precariousness has come to be naturalized as a consequence of the imposition of rules of the game by the hegemonic areas, to which we must now add the consequences resulting from the pandemic caused by the covid-19 and which only will help to further accentuate the gap between academic areas. In the framework of a critical pedagogy and with the help of the theory of the performative interpreted from an educational position, the work reflects on the causes of this situation with the aim of highlighting the tensions to which Spanish Music Education is subjected, preventing its progress in the context of the European Higher Education Area. For this, it is urgent to recognize and accept the differences and imagine other ways of thinking and doing university from the diversity of the areas of knowledge and not from the imposition of current hegemonic thought. It is the intention of this work to incite scientific debate from an area that is intended to banish to the outskirts of knowledge as a consequence of segregating educational policies.
\end{abstract}

Key words: Music education; music teacher training; precariousness; European Higher Education Area.

@José Antonio Rodríguez-Quiles. The content of this article is the sole responsibility of the authors. The Revista Electrónica de LEEME and Universitat de València are not liable for any legal actions that may arise involving the article's content. Revista Electrónica de LEEME - Lista Electrónica Europea de Música en la Educaciónhttp://ojs.uv.es/index/php/LEEME/index ISSN: 1575-9563. Editores: Universidad de Valencia y Jesús Tejada. Visibilidad de esta revista: SCOPUS, Emerging Sources Citation Index (Clarivate), EBSCO, CINDOC (CSIC), Citefactor, COPAC, Dialnet, DICE (CSIC), DOAJ, e-revistas (CSIC), EBSCO Premier, ERIH+, Gale Cengage Learning, IN-RECS, IRESIE, LATINDEX, MIAR, OCLC Worldcat, RESH, REDIB, RILM Core Journals, SUDOC, ULRICHS. Esta revista es de acceso libre mediante licencia Creative Commons 4.0 CC by. Política de archivado: etiqueta verde SHERPA-ROMEO. 


\title{
1. La universidad y su carácter universal
}

\begin{abstract}
El primer tijeretazo a los presupuestos de la Junta de Andalucía por la crisis del coronavirus se lo ha llevado la educación. En concreto las universidades, cuya financiación lleva años padeciendo recortes. Diario El País $(22.05 .20)^{1}$
\end{abstract}

Las palabras universidad, universo y universal presentan la misma etimología. Las tres contienen el término latino unus que hace referencia a algo que no admite división. Estos tres términos aluden así a una multitud de cosas distintas, pero a la vez unidas. Sin embargo, este carácter integrador (y también democrático) de la universitas como institución orientada hacia una meta común (integración de saberes y acciones) es puesto en cuestión por la universidad española de nuestros días. En efecto, aunque las buenas formas de la corrección política aconsejan no invocarlo explícitamente, lo cierto es que de facto existen áreas de conocimiento que quedan desplazadas a los márgenes, cuando no directamente excluidas por la epistemocracia ${ }^{2}$ ejercida por la academia; esto es, por las relaciones de poder que imponen unos campos del saber sobre otros. En este sentido, se hace imprescindible considerar qué se entiende hoy en día por universidad y cómo cambia ese sentido según sea pronunciado por unos u otros representantes de los diferentes saberes y de las diversas acciones. Al igual que ocurre con otras formas de exclusión en la vida pública, esta epistemocracia actúa de modo performativo en el seno de la academia y de instituciones como la Agencia Nacional de Evaluación de la Calidad y Acreditación (ANECA), entre otras; de suerte que, aunque sus acciones no se verbalicen, las consecuencias no dejan margen a la duda. Un caso paradigmático de este hecho en el seno de la universidad española viene representado por el sometimiento de aquellos ámbitos de la música y del hacer musical que se alejan de la contemplación histórica del objeto de estudio. Entre los primeros se situarían la Educación Musical (EM) y las diversas variantes de performance ${ }^{3}$ de los Conservatorios de Música y de Danza, mientras que en el otro extremo estaría el caso de la Musicología y de la Etnomusicología.

El caso de la EM podría muy bien extrapolarse a otras de las denominadas Didácticas Específicas, en contraposición a la Didáctica General, por un lado, y a las áreas que podrían denominarse madres, por el otro. Así, a modo de ejemplos, la Didáctica de las Ciencias Experimentales se encuentra en una encrucijada entre la Didáctica General y las diversas ramificaciones de la Física, la Química, la Biología o la Geología, entre otras. Mientras que la Didáctica de las Ciencias Sociales mantiene un difícil equilibrio entre la Didáctica General y disciplinas como la Geografía, la Historia, la Historia del Arte, etc. En este trabajo, se planteará un análisis desde y para el ámbito musical, si bien no conviene perder de vista que el poder

\footnotetext{
${ }^{1} \mathrm{https} / / /$ tinyurl.com/yan5oq8w

${ }^{2}$ Del griego epistémē (conocimiento) y -krátos (gobierno, poder).

${ }^{3}$ Se usa aquí performance en sentido amplio para incluir todo tipo de interpretación artística con participación de cuerpo e instrumentos musicales, incluyendo obras escritas o improvisadas de cualquier época, género o estilo.
} 
performativo de la epistemocracia se sitúa en el centro de una esfera que extiende su sombra en todas direcciones y no solamente sobre las áreas mencionadas. El objetivo último no es sino justificar que el acercamiento a cualquier aspecto de la música (histórico, performático, creativo, educativo...) con la intencionalidad de profundizar en él y extender su horizonte hacia lo que hoy en día se da en llamar fronteras del conocimiento es también una actividad superior y por tanto debe tener su espacio en la institución universidad; institución que, por otra parte, no deja de definirse como democrática, hoy más que nunca. Urge repensar pues la posición de la Educación Musical y de los Conservatorios españoles en el contexto de la Unión Europea.

En un mundo neoliberal como el nuestro, la economía es la que define en primera y última instancia los servicios fundamentales, incluyendo -claro está- la educación en todos sus niveles. Como consecuencia de esto, ciertas áreas curriculares y académicas son consideradas como prescindibles y acaban relegándose a los márgenes. En este sentido, resulta paradigmática la supresión en España del carácter obligatorio de la música en el currículo para Educación Primaria y Secundaria con la entrada en vigor de la Ley Orgánica 8/2013, de 9 de diciembre, para la mejora de la calidad educativa (LOMCE) (Jefatura del Estado, 2013) y la subsecuente eliminación de la titulación universitaria que antes estuviera expresamente orientada a la formación inicial del profesorado de esta materia. Esta merma curricular resulta un hecho absolutamente insólito en el contexto de la Unión Europea; merma que se entiende menos aún tras los innumerables estudios provenientes del ámbito de la neurociencia (Chobert y Besson, 2012; Habib y Besson, 2009; Levitin y Tirovolas, 2009; Moreno, Marques, Santos, Santos, Castro y Besson, 2009; Peretz y Zatorre, 2003; Tierney y Kraus, 2013; Wan y Schlaug, 2010; Zatorre, Belin y Penhune, 2002) demostrando sin ningún género de dudas la utilidad de una práctica musical continuada y, en consecuencia, la pertinencia de la música como parte insustituible del currículo escolar. Conviene subrayar este carácter único y diferenciador de la música en su doble vertiente como arte y como ciencia que la hacen irreemplazable por ninguna otra materia (Bidal-Loton y Leveau, 2019; Habib, 2019; Ticker, 2017), a lo que debemos sumar la importancia que viene adquiriendo la música en las tareas de reeducación de alumnado con necesidades educativas especiales (Bedoin, Brisseau, Molinier, Roch y Tillmann, 2016; Frey, François, Chobert, Velay, Habib y Besson, 2019; Habib y Commeiras, 2014; Habib, Lardy, Desiles, Commeiras, Chobert y Besson, 2016; Welch, Ockelford, Carter, Zimmermann y Himonides, 2009).

Una vez más, los presupuestos neoliberales -actuando en contra del principio de educación integral para la ciudadanía europea, tal y como recogen los preámbulos de todas las leyes educativas de la Unión Europea sin excepción ${ }^{4}$ - son quienes determinan en última instancia qué áreas hay que proteger y cuáles pueden excluirse. Un problema asociado a este proceder consiste en hacer creer a la sociedad que las áreas "poco productivas" no merecen la atención de

\footnotetext{
${ }^{4}$ Véase Proyecto MeNet en http://menet.mdw.ac.at/menetsite/english/index.html
} http://ojs.uv.es/index/php/LEEME/index ISSN: 1575-9563. Editores: Universidad de Valencia y Jesús Tejada. Visibilidad de esta revista: SCOPUS, Emerging Sources Citation Index (Clarivate), EBSCO, CINDOC (CSIC), Citefactor, COPAC, Dialnet, DICE (CSIC), DOAJ, e-revistas (CSIC), EBSCO Premier, ERIH+, Gale Cengage Learning, IN-RECS, IRESIE, LATINDEX, MIAR, OCLC Worldcat, RESH, REDIB, RILM Core Journals, SUDOC, ULRICHS. Esta revista es de acceso libre mediante licencia Creative Commons 4.0 CC by. Política de archivado: etiqueta verde SHERPA-ROMEO. 
los gobiernos y, peor aún, que aquellos que no son capaces de defender su área merecen ser excluidos, siendo además responsables de su propia exclusión, cuando la realidad es justo la contraria. En efecto, situar la causa de exclusión en las particularidades del área acosada por ser diferente a otras hegemónicas hace recaer la responsabilidad sobre el dominado y no sobre el dominador, si bien esto se hace a veces de formas tan sutiles que consiguen abducir incluso a los propios afectados.

Como expone López (2019) refiriéndose al futuro de las Humanidades en un mundo neoliberal, "[h] ay una consecuencia perversa asociada, más grave si cabe: la progresiva extensión de un tipo de racionalidad que no acepta la existencia de ninguna otra y las expulsa como excrecencia" (p.93). Racionalidad ajena a la advertencia de autoras como Cristina Morales (2019), quien recuerda que "[e]n el extrarradio del progreso también se articula pensamiento, se escribe y se aplica, y si una no es una niñata occidental, sabrá encontrar la potencia aglutinadora que se proyecta desde el suburbio de origen hasta estos otros suburbios nuestros" (p.26). Sin embargo, a juicio del legislador, diríase que la actividad musical en nada contribuye, verbigracia, a la adquisición y desarrollo de competencias transversales, de habilidades comunicativas, confianza individual, entusiasmo, constancia, aceptación del cambio, creatividad, potenciación del pensamiento crítico... y tantas otras.

"Las habilidades cognitivas, siendo imprescindibles, no son suficientes; es necesario adquirir desde edades tempranas competencias transversales, como el pensamiento crítico, la gestión de la diversidad, la creatividad o la capacidad de comunicar, y actitudes clave como la confianza individual, el entusiasmo, la constancia y la aceptación del cambio" (Jefatura del Estado, 2013, p.97860)

Las consecuencias de este pensamiento neoliberal se están padeciendo de forma clara en los últimos años cuando, desde las administraciones educativas tanto central como autonómicas, se ve con buenos ojos y se potencia la creación de escuelas de música privadas no solo de nivel elemental, sino también de niveles medio y superior, así como la apuesta por todo tipo de másteres en universidades públicas y privadas, mientras simultáneamente se suprime la EM del currículo obligatorio y se acaba aminorando la carga lectiva específica de la formación inicial del profesorado de música en las universidades. Así, docentes e investigadores de esta área de conocimiento se sienten cada vez más aislados en el contexto de una institución que les ha arrebatado su propia identidad profesional, generándoles una realidad de fracaso impuesto difícil de asimilar. Parece lógico pensar que, sin una lucha conjunta a nivel nacional (y, por extensión, internacional) entre todos los afectados por esta situación sobrevenida, las posibilidades de cambio no son efectivas. Debemos, no obstante, estar alerta aquí ante la pregunta que se hace Butler (2017) a propósito de los colectivos sociales desfavorecidos. ¿Qué significa actuar unidos cuando las condiciones para la acción conjunta han quedado menoscabadas? En el caso que ocupa este artículo, la artificialidad de muchas de las leyes y normas vigentes sitúan a la Musicología y a la EM en campos académicos separados (Arte y Humanidades, por un lado; Ciencias Sociales

@José Antonio Rodríguez-Quiles. The content of this article is the sole responsibility of the authors. The Revista Electrónica de LEEME and Universitat de València are not liable for any legal actions that may arise involving the article's content. Revista Electrónica de LEEME - Lista Electrónica Europea de Música en la Educación-. http://ojs.uv.es/index/php/LEEME/index ISSN: 1575-9563. Editores: Universidad de Valencia y Jesús Tejada. Visibilidad de esta revista: SCOPUS, Emerging Sources Citation Index (Clarivate), EBSCO, CINDOC (CSIC), Citefactor, COPAC, Dialnet, DICE (CSIC), DOAJ, e-revistas (CSIC), EBSCO Premier, ERIH+, Gale Cengage Learning, IN-RECS, IRESIE, LATINDEX, MIAR, OCLC Worldcat, RESH, REDIB, RILM Core Journals, SUDOC, ULRICHS. Esta revista es de acceso libre mediante licencia Creative Commons 4.0 CC by. Política de archivado: etiqueta verde SHERPA-ROMEO. 
y Jurídicas, por otro), a la par que continúan excluyendo cualesquiera de los estudios históricamente ubicados en los Conservatorios de Música y en los Conservatorios de Danza, creando así la falsa idea de que no existe relación entre unas áreas y otras.

\section{Sobre el carácter ontológico de las áreas de conocimiento}

En el mundo actual en el que nos desenvolvemos, el ser de un área de conocimiento es un ser en relación a las demás áreas en un marco académico. Es decir, un área no puede entenderse como una pieza independiente del gran puzle que la propia taxonomía de la UNESCO ha establecido y a la cual estamos obligados a remitirnos, nos guste o no. Pero ¿qué ocurre cuando uno ni siquiera se ve reflejado, cuando no es considerado, cuando se le ignora mientras que otras áreas aparecen minuciosamente categorizadas e incluso subcategorizadas por esta misma organización internacional $?^{5}$ Dicho de otro modo, se quiera o no, un área de conocimiento está puesta en relación con normas, con organizaciones sociales y políticas que se han desarrollado históricamente con el fin de maximizar la precariedad para unas áreas (p.ej. EM, Humanidades en general...) y de minimizar la precariedad para otras (áreas Físico-Naturales, Tecnológicas, de la Salud...). Desde este punto de vista, no es posible definir la ontología de un área y después ver qué significaciones académicas y sociales presenta. Antes, al contrario, el ser de un área de conocimiento es estar expuesta a ajustes y reajustes dentro y fuera de un marco académico. Dicho de otro modo, la ontología de un área es una ontología de carácter social, expuesta a fuerzas sociales políticamente articuladas, así como a ciertas exigencias de tipo socio-académico: utilidad social, contribución al avance científico, número de artículos de impacto publicados, número de doctores en el seno del área, suma total del número de tramos de investigación reconocidos por la ANECA $^{6}$ entre los profesores del área... Y todo esto, por el empeño de algunos por doblegarnos a un modelo -el anglosajón- que no representa las particularidades españolas.

"En España deberíamos seguir un modelo universitario más mediterráneo, más continental y no tan anglosajón. El modelo que se está imponiendo es un modelo basado en criterios anglosajones y no se adapta tan bien a los países continentales. El propio modelo de Bolonia es un modelo anglosajón que no tiene en cuenta las características y la idiosincrasia de las universidades españolas" (De Faramiñan, 2017). ${ }^{7}$

Si se toma como ejemplo el caso que ocupa este artículo de la EM (oficialmente en España, Área de Didáctica de la Expresión Musical) es evidente el modelado, el proceso de ajustes y reajustes al que el contexto académico-social la viene sometiendo. Así, pasó de ser un área sin

\footnotetext{
${ }^{5}$ La nomenclatura internacional para los campos de Ciencia y Tecnología que la UNESCO divide en campos, disciplinas y subdisciplinas puede consultarse en https://tinyurl.com/ybdnsmfb

${ }^{6}$ Se conoce como tramo o sexenio de investigación un período investigador de seis años que pueden ser consecutivos o no y de cuyo reconocimiento se encarga en España la Agencia Nacional de Evaluación de la Calidad y Acreditación. Por su parte, la Comisión Nacional Evaluadora de la Actividad Investigadora establece anualmente los indicios de calidad y criterios de evaluación de las publicaciones científicas, específicos para cada campo y rama del conocimiento.

${ }^{7}$ https://tinyurl.com/y6uyao2n
} 
pena ni gloria, marginal, a transformarse en la última década del pasado siglo en un área emergente a partir de la puesta en marcha del Título de Maestro en Educación Musical, para pocos años después ser condenada al olvido como consecuencia de la errónea (léase, interesada) interpretación del Plan Bolonia por las autoridades españolas y aumentando con ello la brecha respecto a los países más avanzados en esta área. Se estaría así ante la paradoja consistente en que mientras el ser interno de la EM se ha fortalecido grandemente en los últimos años (si se atiende, por ejemplo, al número de artículos publicados en revistas de impacto), su ontología social en relación al área universitaria de Música (por tomar aquí como ejemplo un área afín) se ha degradado considerablemente.

No debe olvidarse que la propia enunciación de una categoría (centro bilingüe, área emergente, maestro generalista, campus de excelencia ...) construye realidad. De la reiteración de estos eufemismos, surge una relación performativa (no ontológica) entre categoría y realidad que transforma las diferencias en desigualdad. Tómese como ejemplo la proliferación de centros bilingües que se ha producido en España en los últimos años: no sólo en las fachadas de cada vez más colegios de Educación Primaria e institutos de Enseñanza Secundaria, sino igualmente también en las de algunos Conservatorios, puede encontrarse el indicativo que hace alusión a la categoría centro bilingüe, de suerte que su propia enunciación alerta de una realidad diferenciadora entre centros 'bilingües' y centros 'normales'. A partir de ese momento, un centro bilingüe adquiere un plus que le otorgará ciertos beneficios (también económicos) por parte de la administración educativa y le imprimirá una determinada imagen con la que poder venderse a la sociedad. Sin entrar aquí en la realidad interna de esos centros bilingües ni valorar el grado de bilingüismo que de facto presentan, el solo etiquetado actúa performativamente en la mente colectiva en detrimento de los colegios no bilingües, a los que se sitúa en los márgenes de lo que supuestamente debe ser. Esto produce desigualdad y hace creer subrepticiamente que un centro 'normal' no puede ya ofrecer una calidad pedagógica de alto nivel porque - por poner un ejemplola clase de música (y lo mismo es válido con el resto de materias curriculares) no se imparta en inglés, cuando el idioma de impartición poco tiene que ver con el carácter ontológico de esta área (ni de otras), así como tampoco con las necesidades socio-educativas de determinados grupos de población. De este modo, se corre el riesgo de aumentar las desigualdades hacia estos grupos particulares. En suma: si bien centros bilingües y no bilingües pueden sin problema formar parte de categorías diferentes, esta diferencia no debería transformarse en desigualdad de unos respecto a otros.

Volviendo al caso de las áreas de conocimiento en el contexto universitario, deben cuestionarse este tipo de categorizaciones impuestas desde arriba porque nunca son neutrales, sino que se ofrecen como técnicas de dominación de las áreas hegemónicas. ¿Qué implicaciones sociales y académicas tiene ser un área emergente en época del coronavirus? ¿Qué supone ser un 
área afín y respecto a qué? ¿Qué significa para la EM pertenecer a un campus de excelencia tras haber quedado reducida a su mínima expresión?...

En efecto, las normas tienen un carácter performativo que desde las áreas hegemónicas construye categorías a través de reiteraciones, penalizaciones, censuras... y que por medio de estas sanciones se 'enseña' cómo actuar; actuación que muchas veces implica el alejamiento de la propia esencia de la EM, que va quedando cada vez más desfigurada. Así, mientras el Ministerio de Educación crea por primera vez un comité de matemáticos para rediseñar la asignatura en tiempos de la covid-198, algunas Comunidades Autónomas proponen que el profesorado de Música dedique al menos parte de su tiempo a impartir materias troncales ${ }^{9}$. No se trata pues de admitir condescendientemente y para tapar bocas ciertas áreas incompletas, acientíficas, improductivas... sino de facilitar espacios académicos en donde todas las áreas (con sus luchas e historias particulares) puedan ser reconocidas, sin separar maquiavélicamente entre lo que la hegemonía considera legítimo, cientifico, aceptable, económicamente productivo... y lo que esta misma hegemonía interpreta como accesorio, prescindible, decorativo, ocioso..., en fin: improductivo.

\section{El triángulo Edípico-Musical}

Mientras la EM, en la mayor parte de los países del centro, Norte y Este de Europa, consiguió a lo largo de la segunda mitad del siglo pasado independizarse poco a poco de otras áreas de conocimiento a las que estaba ligada para constituirse como área con identidad propia en el ámbito de las enseñanzas superiores -siendo el caso alemán paradigmático en este sentido (Gruhn, 2003; Kruck, 2013) - la situación de los países del Sur de Europa ha corrido peor suerte (Liimets y Maeesalu, 2011; Malmberg y Wimmer, 2007). Tomando como metáfora el conocido mito de Edipo, la EM española - como área académica-podría situarse en el centro de un triángulo sobre el que actúan fuerzas de distinto signo conformando un marco que la constriñe. Como, además, la propia área se nutre recurrentemente de profesionales formados en primera instancia en los ámbitos de la Musicología, la interpretación instrumental (Conservatorios) o la Pedagogía General, se corre el riesgo de concebir éstas cómo 'áreas madre', con el peligro que supone -a modo de complejo de Edipo- conceptualizar la EM desde supuestos epistemológicos que no le son propios.

\footnotetext{
${ }^{8} \mathrm{https} / / /$ tinyurl.com/y9wtrc8b

9 https://tinyurl.com/y9ugennd
} 

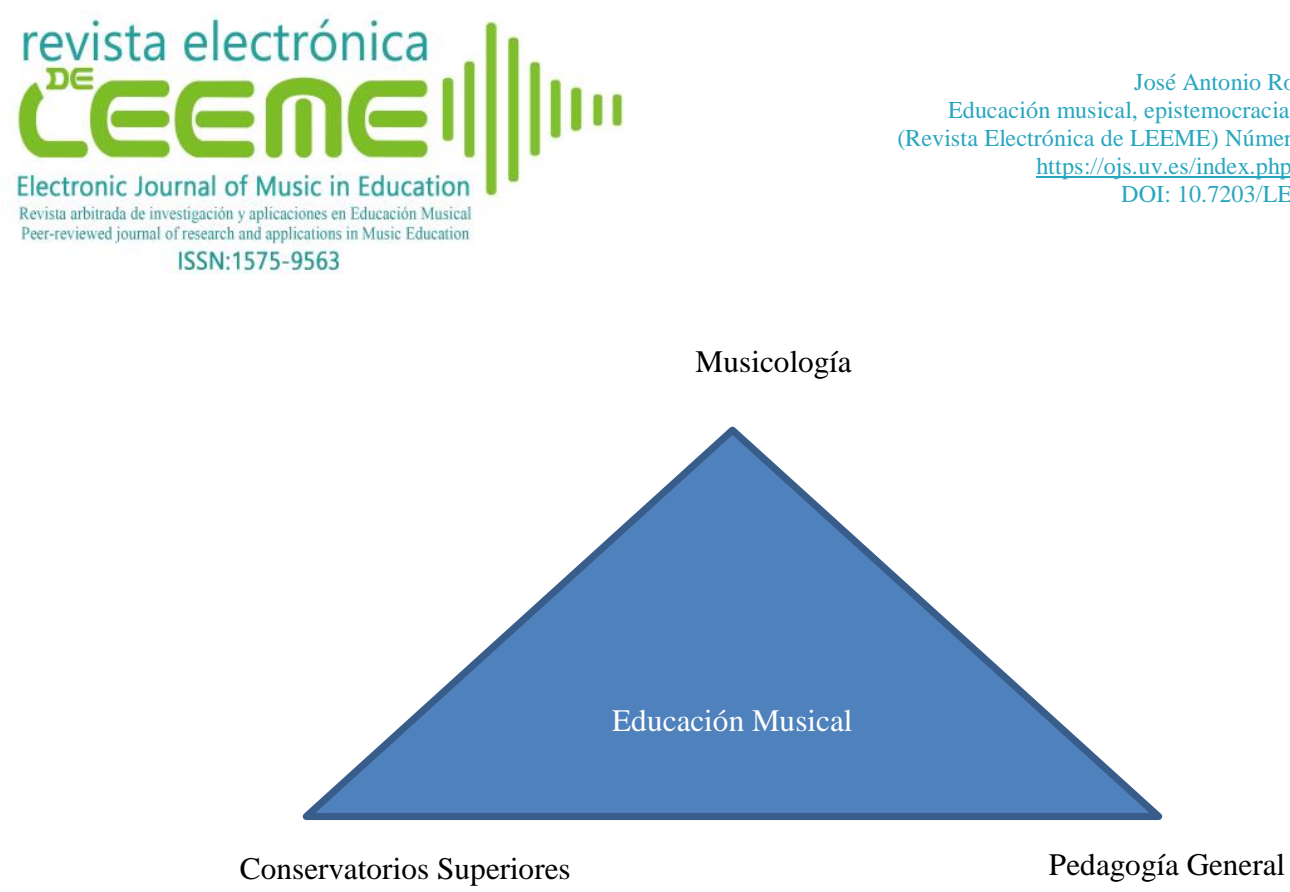

Figura 1. Triángulo Edípico-Musical

El debate sobre maestro especialista versus maestro generalista representa, por cierto, un dilema mal planteado. La etiqueta que se le ponga al profesional encargado de una educación integral a través de la música es algo secundario. Lo verdaderamente importante es preguntarse por la formación musical, pedagógica y didáctica con la que este profesional cuenta para afrontar su labor docente (e investigadora). En este sentido, España falla en absolutamente todos los aspectos relacionados con la formación inicial del profesorado de EM y en todos los niveles: Educación Infantil, Primaria, Secundaria, pero también en la formación del futuro profesorado de instrumento. La constatación es tan fácil como echar un vistazo a los planes de estudios de nuestros vecinos del centro, Norte y Este de Europa y comprobar las amplias diferencias existentes.

Por otra parte, la creación de una Mención en Educación Musical como parte de los estudios generalistas del Grado de Maestro en Educación Primaria, en sustitución del extinto Título de Maestro en Educación Musical, lejos de ser una concesión graciosa al área por parte de la universidad española, se presenta como una estrategia política que consiste en crear al Otro para poder marginarlo mejor. Así, desde el momento en que comenzaron a salir de las universidades los actuales 'especialistas' en $\mathrm{EM}^{10}$ la sociedad dispone ya de argumentos más que suficientes para -si quisiera- poder mofarse de su deficiente preparación, el área quedará más devaluada aún en los próximos años y, en consecuencia, se tendrán recursos dialécticos para

\footnotetext{
${ }^{10}$ Recuérdese que la 'especialización', hasta la llegada de la pandemia por la Covid-19, venía durando unas escasas trece semanas de clases presenciales y que el alumnado que a ella accede no tiene que demostrar relación previa con la música de manera activa, ni conocimientos de Lenguaje Musical, ni dominio de instrumento alguno. Salvo en algunas universidades que cuentan con prueba de selección específica como la Universidad de Valencia, por ejemplo.
}

@ José Antonio Rodríguez-Quiles. The content of this article is the sole responsibility of the authors. The Revista Electrónica de LEEME and Universitat de València are not liable for any legal actions that may arise involving the article's content. Revista Electrónica de LEEME - Lista Electrónica Europea de Música en la Educación-. http://ojs.uv.es/index/php/LEEME/index ISSN: 1575-9563. Editores: Universidad de Valencia y Jesús Tejada. Visibilidad de esta revista: SCOPUS, Emerging Sources Citation Index (Clarivate), EBSCO, CINDOC (CSIC), Citefactor, COPAC, Dialnet, DICE (CSIC), DOAJ, e-revistas (CSIC), EBSCO Premier, ERIH+, Gale Cengage Learning, IN-RECS, IRESIE, LATINDEX, MIAR, OCLC Worldcat, RESH, REDIB, RILM Core Journals, SUDOC, ULRICHS. Esta revista es de acceso libre mediante licencia Creative Commons 4.0 CC by. Política de archivado: etiqueta verde SHERPA-ROMEO. 
marginarla mucho más de lo que está ahora, desde el momento en que el triángulo EdípicoMusical irá creciendo en favor de las tres madres, quedando la EM a su sombra (Figura 2).

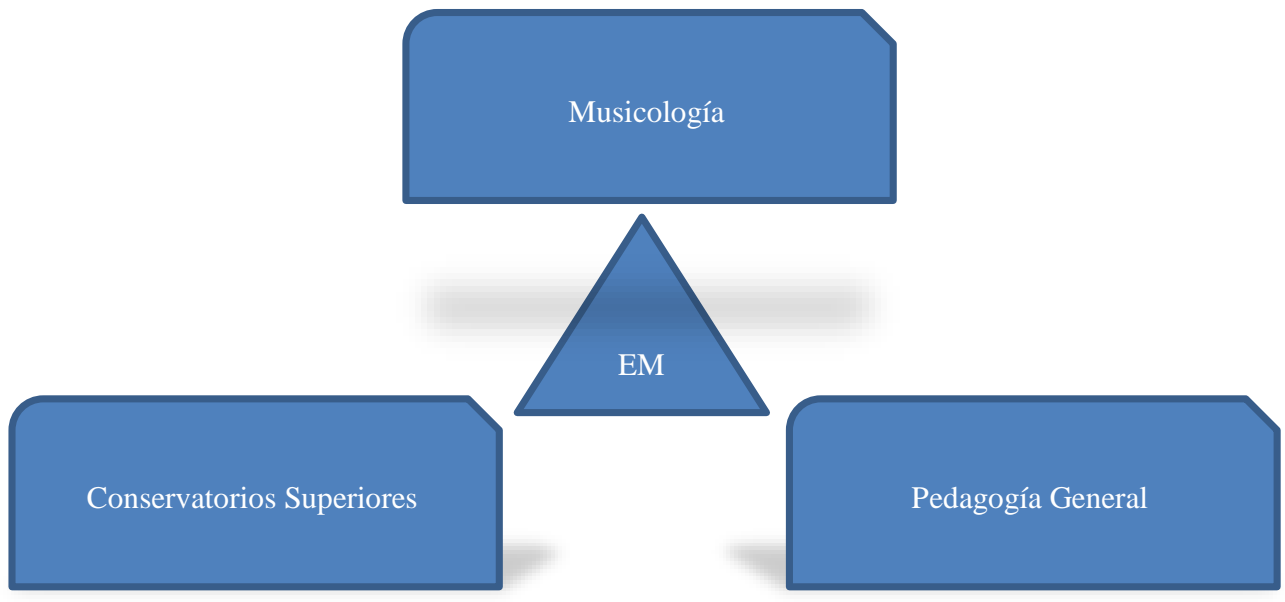

Figura 2. A la sombra del triángulo Edípico-Musical

En lugar de darlos como correctos, se hace necesario pues criticar los procedimientos por los que se jerarquizan las diferentes áreas y campos de conocimiento, puesto que en la misma jerarquía aparece ya el sometimiento. El caso de las Facultades de Educación resulta paradigmático en este sentido. Incluso el alumnado menos avezado es capaz de detectar con facilidad qué departamentos controlan la propia estructura medular de estos centros y qué áreas aparecen como comparsas en el conjunto. ¿Por qué hacer encajar a todas las áreas en un sistema creado por unos pocos y para que esos pocos mantengan sus privilegios? Dentro de las áreas desfavorecidas, a aquellos docentes que han conseguido posicionarse (normalizarse) en el sistema, ¿se les debe exigir algo? La pregunta no es retórica, puesto que como ya advirtiera Mouffe (2007, p.25)): "[T] odo orden hegemónico es susceptible de ser desafiado por prácticas contrahegemónicas, es decir, prácticas que van a intentar desarticular el orden existente para instaurar otra forma de hegemonía". Dicho de otro modo, habrá que estar alerta para evitar que, además, estas personas de áreas desfavorecidas impongan sus particulares criterios normalizadores implantando una única forma de organización interna, decidiendo en qué dirección única se debe avanzar, privilegiando a unos colegas en detrimento de otros...

\section{Precariedad y educación musical}

Judith Butler usa el término precaridad para designar 


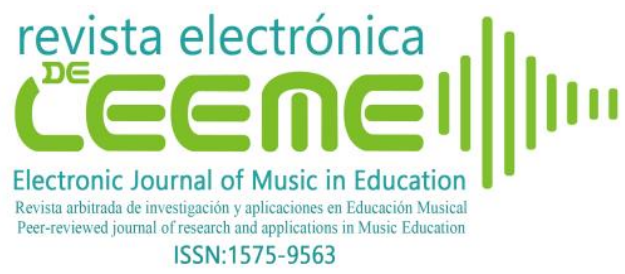

"[u]na condición impuesta políticamente merced a la cual ciertos grupos de la población sufren la quiebra de las redes sociales y económicas de apoyo mucho más que otros, y en consecuencia están más expuestos a los daños, la violencia y la muerte" (Butler, 2017, p.40).

De este modo, la precaridad no es más que la distribución diferenciada de la precariedad. Estas consecuencias manifestadas en forma de daño, violencia e, incluso, muerte a las que alude la filósofa americana se dan igualmente en el seno de la academia en ciertas áreas de conocimiento, si bien de formas sutiles que pasan desapercibidas para los no directamente afectados. Esto es así porque la institución Universidad no reconoce de la misma forma a todas las áreas de conocimiento que la configuran ni establece ningún tipo de medidas de apoyo para las más vulnerables. Este tratamiento de precaridad académica conduce a situaciones paradójicas si se piensa en el ámbito musical. Así, por ejemplo, presumir de contar con la orquesta de la universidad, pero constituida por músicos ajenos a ella, la inmensa mayoría tomados prestados de los Conservatorios y a quienes, por otro lado, no reconoce como dignos para abrazar de pleno derecho la institución que los necesita para determinados fines. O algunos docentes del Área de EM que prefieren integrarse en proyectos de otras áreas de conocimiento porque les pueden otorgar mayor prestigio, pero sobre todo mayores facilidades a la hora de hacer carrera universitaria, aun cuando esos proyectos no estén relacionados directamente con el área que representan.

Para poder entender por qué determinadas áreas (performance, composición, dirección orquestal...) no son reconocidas por la universidad española, así como para comprender el reconocimiento fingido de otras áreas universitarias (léase, EM), resulta aconsejable acercarse a los modos de existencia de sus miembros y captar el sentido que para ellos tienen los conceptos educación superior e investigación. Por ejemplo, es necesario preguntarse sobre los modos en que entiende la docencia uno-a-uno un docente de canto o de instrumento, o en dónde sitúa las fronteras del conocimiento un compositor de vanguardia; pero, también, cómo el profesorado universitario de EM se ve obligado a redefinir un programa de estudio para orientarlo a una misión imposible: capacitar como docentes de Música competitivos a nivel europeo (según los requerimientos del Plan Bolonia) y en tiempo récord (solo 6 ECTS como única materia obligatoria en los estudios de Grado tanto para educación Primaria como para Secundaria y Bachillerato) a un alumnado que a duras penas entiende el sentido de una tal materia descontextualizada en su plan de estudios.

Por si esta situación no fuese alarmante por sí misma, es fácil pronosticar que la inesperada irrupción de la pandemia causada por la covid-19 no servirá más que para seguir aumentando la brecha entre materias de primera y de segunda, ahondando así en el drama de una EM que podría tornarse virtual, no solo en el sentido de materia online, sino también en la acepción que la RAE concede a este adjetivo como algo que tiene existencia aparente y no real. Nuevamente, la música se aparece como mero elemento decorativo del currículo en todas las

@José Antonio Rodríguez-Quiles. The content of this article is the sole responsibility of the authors. The Revista Electrónica de LEEME and Universitat de València are not liable for any legal actions that may arise involving the article's content. Revista Electrónica de LEEME - Lista Electrónica Europea de Música en la Educación-. http://ojs.uv.es/index/php/LEEME/index ISSN: 1575-9563. Editores: Universidad de Valencia y Jesús Tejada. Visibilidad de esta revista: SCOPUS, Emerging Sources Citation Index (Clarivate), EBSCO, CINDOC (CSIC), Citefactor, COPAC, Dialnet, DICE (CSIC), DOAJ, e-revistas (CSIC), EBSCO Premier, ERIH+, Gale Cengage Learning, IN-RECS, IRESIE, LATINDEX, MIAR, OCLC Worldcat, RESH, REDIB, RILM Core Journals, SUDOC, ULRICHS. Esta revista es de acceso libre mediante licencia Creative Commons 4.0 CC by. Política de archivado: etiqueta verde SHERPA-ROMEO. 
etapas educativas. Una de las consecuencias más preocupantes de esta involución tendrá que ver con la eliminación de los procesos estético-educativos emergentes propios de los actos performativos facilitados por la co-presencia (física) entre todos los implicados en el proceso de enseñanza-aprendizaje musical, quedando con ello perturbadas -por ejemplo- parte de las características que definen una educación musical performativa (Gould, 2012; Ramírez y Rodríguez-Quiles, 2020; Rodríguez-Quiles 2017, 2018), justo las que afectan directamente a la parte más humana de la persona en su relación con sus semejantes y que no pueden de ningún modo pretender ser sustituidos por sucedáneos virtuales sin que se altere la esencia misma del ser persona. En la misma línea, la socióloga canadiense Sarah Thornton (2020), arguye:

\footnotetext{
"Al contrario de lo que ocurre con películas y libros, el Arte no puede ser pasado al formato digital sin que pierda su esencia. El Arte con mayúsculas no trata solo de la vista y los ojos; es una experiencia física que te sacude por dentro" (p.1). ${ }^{11}$
}

Es posible que, aprovechando el precedente debido al estado de contingencia impuesto por la Covid-19, la idea de una virtualización generalizada de la educación sea justamente la que seduzca a más de un gestor de las políticas educativas de nuestro país por motivos económicos más que sanitarios, obviando que existen aspectos de la educación que, siendo intrínsecamente humanos, no pueden sustituirse por alternativas virtuales. Hay aquí que recordar que las autoridades alemanas de los ámbitos de la cultura y la educación han defendido la EM, incluso en los tiempos más críticos de la pandemia:

\begin{abstract}
"Hasta nuevo aviso, todas las clases tendrán lugar de forma virtual [...] a excepción de algunas prácticas de laboratorio y cursos de Música, siempre que se cumplan las dimensiones espaciales adecuadas" (Vicerrector de Ordenación Académica de la Universidad de Potsdam, circular a la comunidad universitaria, 24 de abril de 2020).
\end{abstract}

Y es que, el reconocimiento académico de un área de conocimiento va ligado a que exista una forma de presentación para esta en el seno de una comunidad científica, altamente globalizada hoy en día. Pero, ¿cuál ha sido tradicionalmente la forma de presentación de la EM? En España, y por reducir aquí por falta de espacio solo al ámbito universitario, este modo de presentación viene siendo regulado en los últimos años a través de la ANECA, imponiendo unas condiciones cada vez más estrictas para la acreditación del profesorado universitario en sus diversas modalidades y sin tener en cuenta las especificidades de determinadas áreas a las que se les obliga a competir en condiciones altamente desfavorables, forzando con ello a desdibujar en mayor o menor medida la esencia de estas áreas como consecuencia lógica de la desnaturalización profesional de quienes las representan. Cada vez es menor el profesorado universitario de los Departamentos de Musicología y de EM que hace música de manera regular como algo consustancial a su profesión, ya que esto resulta incompatible con la dedicación a tiempo completo que exige la investigación y publicación en revistas de alto impacto. Estas revistas

\footnotetext{
${ }^{11}$ https://tinyurl.com/ydx32vsj
} 
específicas en EM escasean a día de hoy y por lo mismo la competencia resulta comparativamente más alta aún que en aquellas otras áreas consolidadas y con mayores recursos, redes e infraestructuras. Como consecuencia, se está cayendo poco a poco en la grave contradicción de hablar/escribir mucho sobre Educación Musical mientras que, en relación inversa, el profesorado va alejándose de la performance musical, esto es, del propio hacer musical en todas sus amplias manifestaciones. Es por ello que, desde la educación musical performativa, se alerta sobre esta contradicción promoviendo conscientemente una práctica musical educativa y una investigación en esta área de conocimiento que resulten: 1) autorreferenciales, esto es, que tengan que ver directamente con las vidas de sus protagonistas; 2) que sean constituyentes de nuevas realidades tendentes a usar, reformular o incluso transgredir las normas opresoras impuestas por otros, teniendo presente que "[a]unque las normas parecen determinar [lo que puede] aparecer, fallan a la hora de controlar la esfera de aparición" (Butler, 2017, p.45).

Abundando en esta idea sobre la importancia de actuar contra determinadas imposiciones, Butler (2017) advierte sobre las renuncias que implica plegarse a ciertas normas. Esto es justamente la tendencia que se viene observando en la EM española, la cual está condenando al profesorado de esta área a dejar de estar musicalmente vivo, por muchos tramos de investigación que la ANECA les pueda otorgar y por los que pasen a ser académicamente reconocidos, en ese reconocimiento falseado que Butler tan acertadamente condena en otros contextos, pero que en este ámbito resulta igualmente válido. El problema viene cuando este profesorado acepta acríticamente el marco de actuación impuesto (el ideal normativo), haciendo incluso de altavoz de las normas del propio marco que le constriñe pero que llega a naturalizar hasta el punto de hacerlo desaparecer de su foco de atención, renunciando a lo propio y característico de la práctica musical educativa (o reduciéndola a su mínima expresión), cuando no debiera haber una separación maniquea entre la reflexión teórica y la actividad práctica y que, por lo mismo, ambas facetas tendrían que ser reconocidas por la academia en igualdad de condiciones, ya que la una no se entiende sin la otra. Parafraseando a la filósofa americana, en estos casos lo que se llega incluso a poner en cuestión son las condiciones ontológicas de su pervivencia en el seno de la academia, siendo esta (el 'ser' del área) una de las poderosas razones que, desde su perspectiva excluyente, la universidad española arguye para impedir el acceso de los Conservatorios Superiores a la vida universitaria pero también para obstaculizar el desarrollo del área de EM en nuestro país, contraviniendo con ello la filosofía misma del Espacio Europeo de Educación Superior y de Investigación, desde el momento en que nada parecido ocurre en los países de nuestro entorno tradicionalmente avanzados en estos ámbitos académicos. 


\section{A modo de conclusión}

Ante esta situación de precariedad a la que la EM española se ha visto abocada como consecuencia de una epistemocracia inflexible y ante los malos augurios para la época postcovid, se hace urgente, entre otras cosas:

- Reconocer las diferencias e imaginar otras formas de pensar y hacer universidad desde la diversidad de las áreas de conocimiento, no desde lo normativo.

- $\quad$ Poner de manifiesto las relaciones de poder existentes entre la multiplicidad de áreas.

- Superar los cánones normalizadores y el pensamiento hegemónico de la academia, rechazando el estar antes que el ser.

- Tematizar la opresión de la Educación Musical también en nuestras aulas, incluyendo los asuntos aquí tratados en los programas de estudio de los diferentes niveles educativos, en los proyectos de innovación..., buscando puntos de fuga - en el sentido de G. Deleuze- que nos ayuden a salir de lo normativo.

- $\quad$ Repensar el concepto utilidad de la Música, de la Educación Musical, de las áreas de conocimiento...

- Valorar por lo que profesionalmente se es y no por cómo se aproxima uno normativamente a lo que no es para salir adelante.

- $\quad$ Fundamentar la epistemología de la EM con argumentos desde dentro del área y no con relación a otras áreas afines, para lo cual es imprescindible que los profesionales de esta materia se narren sin complejos desde el Yo, tanto individual como colectivo.

El tiempo dirá si la crisis en la cual la Covid-19 ha sumido al país servirá para que los profesionales de la EM en España tomen consciencia de la crítica situación de supervivencia de su área y -unidos- levanten la voz, o si las imposiciones del mercado conseguirán aniquilar definitivamente la propia esencia de la Educación Musical como acto verdaderamente socioeducativo en un mundo en donde los puentes hacia el Otro tendrían que multiplicarse sin cesar y para lo cual la música brinda posibilidades incalculables.

\section{Referencias}

Bedoin, N., Brisseau, L., Molinier, P., Roch, D. y Tillmann, B. (2016). Temporally regular musical primes facilitate subsequent syntax processing in children with Specific 
Language Impairment. Frontiers in Neuroscience, 10, 245. doi:10prg/10.3389/fnins.2016.00245

Bidal-Loton, M. y Leveau, N. (2019). La petite chanson des émotions et de la motivation, en Rodríguez-Quiles, J.A. (Ed.). Bienfaits de la musique à l'école. Une expérience européenne (pp.53-68). Potsdam: UP.

Butler, J. (2017). Cuerpos aliados y lucha política. Hacia una teoría performativa de la asamblea. Barcelona: Paidós.

Chobert, J. y Besson, M. (2012). Influence de l'apprentissage de la musique sur la perception des syllabes chez les enfants normolecteurs et dyslexiques. En M. Habib, E. Bigand y V. Brun (Eds.), Musique et cerveau: nouveaux concepts, nouvelles applications (pp.61-75). Montpellier: Sauramps.

Frey, A., François, C., Chobert, J., Velay, J.L., Habib, M. y Besson, M. (2019). Music Training Positively Influences the Preattentive Perception of Voice Onset Time in Children with Dyslexia: A Longitudinal Study. Brain Science, 9(91), 1-24. doi:10.3390/brainsci9040091

Gould, E. (2012). Moving Responses: Communication and Difference in Performative Pedagogies, Theory Into Practice, 51, 196-203. doi:10.1080/00405841.2012.690303

Gruhn, W. (2003). Geschichte der Musikerziehung. Hofheim: Wolke.

Habib, M. (2019). La musique à l'école: son impact neurologique. En J.A. Rodríguez-Quiles (Ed.), Bienfaits de la musique à l'école. Une expérience européenne (pp.31-52). Potsdam: Universitätsverlag.

Habib, M. y Besson, M. (2009). What do Music Training and Musical Experience Teach Us About Brain Plasticity? Music Perception: An Interdisciplinary Journal, 26(3), 279-285. doi:10.1525/mp.2009.26.3.279

Habib, M. y Commeiras, C. (2014). «Mélodys»: remédiation cognitivo-musicale des troubles de l'apprentissage. Bruxelles: De Boeck.

Habib, M., Lardy, C., Desiles, T., Commeiras, C., Chobert, J. y Besson, M. (2016). Music and Dyslexia: A New Musical Training Method to Improve Reading and Related Disorders. Frontiers in Psychology, 7(26), 1-15. doi:10.3389/fpsyg.2016.00026

Jefatura del Estado (2013). Ley Orgánica 8/2013 para la mejora de la calidad educativa. BOE (10/12/2013), núm.295, referencia 12886, pp.97858-97921. 
Kruck, R. (2013). Das didaktische Problem der subjektiven Irritation. Systemischer Konstruktivismus in aktuellen musikpädagogischen Konzeptionen. Münster: MV Wissenschaft.

Levitin, D. y Tirovolas, A. (2009). Current Advances in the Cognitive Neuroscience of Music. The Year in Cognitive Neuroscience 2009. Annuals of the New York Academy of Science, 1156, 211-231. doi:10.1111/j.1749-6632.2009.04417.x

Liimets, A. y Maeesalu, M. (Eds.) (2011). Music Inside and Outside The School. Baltische Studien zur Erziehungs- und Sozialwissenschaft. Berlin: Peter Lang.

López, J. (2019). Crítica de la razón precaria. La vida intelectual ante la obligación de lo extraordinario. Madrid: Catarata.

Malmberg, I. y Wimmer, C. (2007). Communicating Diversity: Musik lehren und lernen in Europa. Ausburg: Wißner-Verlag.

Morales, C. (2019). Lectura fácil. Barcelona: Anagrama.

Moreno, S., Marques, C., Santos, A., Santos, M., Castro, S. y Besson, M. (2009). Musical training influences linguistic abilities in 8-year-old children: More evidence for brain plasticity. Cerebral Cortex, 19, 712-723. doi:10.1093/cercor/bhn120

Mouffe, Ch. (2007). En torno a lo politico. Buenos Aires: FCE.

Peretz, I. y Zatorre, R. (2003). The cognitive neuroscience of music. Oxford: Universtity Press.

Ramírez, M.F. y Rodríguez-Quiles, J.A. (2020). Educación Musical Performativa en la formación de intérpretes. Un estudio de caso. Revista Electrónica de LEEME, 45, 17-34. doi:10.7203/LEEME.45.16231

Rodríguez-Quiles, J.A. (2017). Rethinking Music Education: Towards a Performative Turn. En J.A. Rodríguez-Quiles (Ed.), Internationale Perspektiven zur Musik(lehrer) ausbildung in Europa (pp.19-37). Postdam: Universitätsverlag Postdam.

Rodríguez-Quiles, J.A. (2018). La música como rizoma. Bases para una educación musical performativa. Revista Musical Chilena, 72(229), 129-150. doi:10.4067/s071627902018000100139

Ticker, C. (2017). Music and the Mind: Music's Healing Powers. Musical Offerings, 8(1), 1-12. doi:10.15385/jmo.2017.8.1.1

@ José Antonio Rodríguez-Quiles. The content of this article is the sole responsibility of the authors. The Revista Electrónica de LEEME and Universitat de València are not liable for any legal actions that may arise involving the article's content. Revista Electrónica de LEEME - Lista Electrónica Europea de Música en la Educaciónhttp://ojs.uv.es/index/php/LEEME/index ISSN: 1575-9563. Editores: Universidad de Valencia y Jesús Tejada. Visibilidad de esta revista: SCOPUS, Emerging Sources Citation Index (Clarivate), EBSCO, CINDOC (CSIC), Citefactor, COPAC, Dialnet, DICE (CSIC), DOAJ, e-revistas (CSIC), EBSCO Premier, ERIH+, Gale Cengage Learning, IN-RECS, IRESIE, LATINDEX, MIAR, OCLC Worldcat, RESH, REDIB, RILM Core Journals, SUDOC, ULRICHS. Esta revista es de acceso libre mediante licencia Creative Commons 4.0 CC by. Política de archivado: etiqueta verde SHERPA-ROMEO. 
Tierney, A. y Kraus, N. (2013). Music training for the development of reading skills. Progress in Brain Research, 207, 209-241. doi:10.1016/B978-0-444-63327-9.00008-4

Wan, C.Y. y Schlaug, G. (2010). Music Making as a Tool for Promoting Brain Plasticity across the Life Span. Neuroscientist, 16(5), 566-577. doi:10.1177/107385410377805

Welch, G., Ockelford, A., Carter, F. Ch., Zimmermann, S.A. y Himonides, E. (2009). Sounds of Intent': mapping musical behaviour and development in children and young people with complex needs, Psychology of Music, 37(3), 348-370. doi:10.1177/0305735608099688

Zatorre, R.J., Belin, P. y Penhune, V.B. (2002). Structure and function of auditory cortex: music and speech. Trends in Cognitive Sciences, 6(1), 37-46. doi:10.1016/S13646613(00)01816-7

@.José Antonio Rodríguez-Quiles. The content of this article is the sole responsibility of the authors. The Revista Electrónica de LEEME and Universitat de València are not liable for any legal actions that may arise involving the article's content. Revista Electrónica de LEEME - Lista Electrónica Europea de Música en la Educación-. http://ojs.uv.es/index/php/LEEME/index ISSN: 1575-9563. Editores: Universidad de Valencia y Jesús Tejada. Visibilidad de esta revista: SCOPUS, Emerging Sources Citation Index (Clarivate), EBSCO, CINDOC (CSIC), Citefactor, COPAC, Dialnet, DICE (CSIC), DOAJ, e-revistas (CSIC), EBSCO Premier, ERIH+, Gale Cengage Learning, IN-RECS, IRESIE, LATINDEX, MIAR, OCLC Worldcat, RESH, REDIB, RILM Core Journals, SUDOC, ULRICHS. Esta revista es de acceso libre mediante licencia Creative Commons 4.0 CC by. Política de archivado: etiqueta verde SHERPA-ROMEO. 